\title{
Downregulation of the Notch signaling pathway inhibits hepatocellular carcinoma cell invasion by inactivation of matrix metalloproteinase-2 and -9 and vascular endothelial growth factor
}

\author{
LIANG ZHOU $^{1,2^{*}}$, DE-SHENG WANG ${ }^{2 *}$, QING-JUN LI ${ }^{2 *}$, WEI SUN ${ }^{2}$, YONG ZHANG ${ }^{2}$ and KE-FENG DOU ${ }^{2}$ \\ ${ }^{1}$ Department of General Surgery, The 155 Central Hospital of PLA, Kaifeng, He'nan 471000; ${ }^{2}$ Department of \\ Hepatobiliary Surgery, Xijing Hospital, The Fourth Military Medical University, Xi'an, Shannxi 710032, P.R. China
}

Received March 27, 2012; Accepted May 25, 2012

DOI: $10.3892 / o r .2012 .1880$

\begin{abstract}
Hepatocellular carcinoma (HCC) is one of the most common malignancies. The main cause of death in HCC patients is tumor progression with invasion and metastasis. However, the underlying mechanisms of HCC invasion and metastasis are still not fully understood. Some studies show that the Notch signaling pathway may participate in tumor invasion and metastasis. However, the mechanisms by which the Notch signaling pathway mediates tumor cell invasion, especially in hepatocellular carcinoma, are not yet known. In the current study, we investigated the anti-invasion effect of the downregulation of the Notch signaling pathway by DAPT in HCC cells. The Notch signaling pathway inhibitor could suppress invasion of HCC cells via the extracellular signalregulated kinases 1 and 2 (ERK1/2) signaling pathways, resulting in the downregulation of matrix metalloproteinase-2 and -9 (MMP-2 and -9) and vascular endothelial growth factor (VEGF). These observations suggested that inhibition of the Notch signaling pathway by DAPT would be useful for devising novel preventive and therapeutic strategies targeting invasion of HCC.
\end{abstract}

\section{Introduction}

Hepatocellular carcinoma (HCC) is the seventh most common malignancy and the third leading cause of cancer-related deaths worldwide (1). Despite improvements in detection and clinical treatment strategies, the 5-year survival rate for HCC is still very low (2). The main cause of death in HCC patients is

Correspondence to: Dr Ke-feng Dou, Department of Hepatobiliary Surgery, Xijing Hospital, The Fourth Military Medical University, Xi'an, Shannxi 710032, P.R. China

E-mail: kefengdou@126.com

*Contributed equally

Key words: Notch signaling pathway, matrix metalloproteinase, vascular endothelial growth factor, extracellular signal-regulated kinase, invasion, hepatocellular carcinoma tumor progression with invasion and metastasis. However, the underlying mechanisms of HCC invasion and metastasis are still not fully understood (3). Thus, the discovery and subsequent development of novel agents to block HCC invasion and metastasis are primary research objectives for HCC.

Tumor metastasis occurs by a series of steps, including cell invasion, degradation of basement membranes, and the stromal extracellular matrix, ultimately leading to tumor cell invasion and metastasis. Matrix metalloproteinases (MMPs) are a family of related enzymes that degrade the extracellular matrix (ECM) and activation of these enzymes allow tumor cells access to the vasculature, migration, and invasion into target organs, and the development of tumor metastasis (4). Among the previously reported human MMPs, MMP-2 and MMP-9 play the most important role in tumor invasion and metastasis because of their specificity for type IV collagen which is the principal component of the basement membrane $(5,6)$. Angiogenesis plays an important role in tumor metastasis from the initial stage of carcinogenesis to the end stage of metastatic disease (7). The development of neovasculature in the tumor provides essential functions for growth, invasion and metastasis. VEGF is one of the isolated angiogenic peptides, and is the most well studied angiogenic factor so far. Moreover, VEGF is known to play a vital role in tumorassociated invasion $(8,9)$.

The Notch signaling pathway includes Notch ligands, receptors, negative and positive modifiers, and Notch target transcription factors. As an important signaling pathway, Notch is not only involved in cell development and fate determination, but also plays an important role in tumor development $(10,11)$. The Notch signaling pathway is aberrantly activated in a variety of human tumors, including T-cell acute lymphoblastic leukemia, lung, colorectal, prostate, and breast carcinomas (12-15). In contrast to its tumor-facilitative role, the Notch signaling pathway has been identified in B-cell malignancies (16), neural crest tumors (17) and skin cancer (18). Therefore, the Notch signaling pathway seems to function as an oncogene or a tumor suppressor, depending on the tissue type. Pharmacologic manipulation of the Notch signaling pathway is becoming a new strategy for human tumors. $\gamma$-secretase inhibitors (GSI) can inhibit the proteolytic processing of Notch receptors by $\gamma$-secretase, which is 
essential for Notch activation (19), and are being investigated clinically in T-cell leukemia and breast cancer.

However, knowledge of the role of the Notch signaling pathway in invasion of $\mathrm{HCC}$ is still limited. Therefore, in this study, we investigated the role and mechanism of Notch signaling pathway inhibition by DAPT. DAPT, a GSI, resulted in inhibiting HCC cells invasion in vitro. Our results suggest that the inhibition of Notch signaling pathway caused decreases of MMP-2, MMP-9 and VEGF, thus resulting in the inhibition of HCC cell invasion, mediated through the inactivation of extracellular signal-regulated kinase (ERK) phosphorylation.

\section{Materials and methods}

Cell culture and reagents. The human liver non-tumor cell line (HL-7702) and the HCC cell lines (HepG2, HuH-7, SMMC7721 and $\mathrm{MHCC} 97 \mathrm{H}$ ) were cultivated in DMEM medium supplemented with $10 \%$ fetal calf serum (Sigma Chemicals Co., St. Louis, MO). The liver non-tumor cell and HCC cells were seeded into 6 -well cell culture plates at a density of $1 \times 10^{5}$ cells/well. All experiments were carried out using confluent cultures. To attain normoxic condition, cultures were maintained at $37^{\circ} \mathrm{C}$ in a humidified incubator containing $20 \% \mathrm{O}_{2}$, $5 \% \mathrm{CO}_{2}$, and $75 \% \mathrm{~N}_{2}$. Primary antibodies for MMP-2, MMP-9 and VEGF were purchased from Santa Cruz Biotechnology (Santa Cruz, CA). Primary antibodies for the Notch1 intracellular domain (NICD) and ERK1/2 were purchased from Abcam (Cambridge, UK). All secondary antibodies were obtained from Pierce (Rockford, IL). MMP-2 small interfering RNA (siRNA), MMP-9 siRNA, VEGF siRNA, and siRNA control were obtained from Santa Cruz Biotechnology. Lipofectamine 2000 was purchased from Invitrogen (Carlsbad, CA, USA). To suppress the Notch signaling pathway, DAPT in DMSO was used at different doses. To inhibit ERK1/2, PD98059 (Calbiochem, San Diego, CA) in DMSO was used at $10 \mu \mathrm{mol} / \mathrm{l}$. All other chemicals and solutions were purchased from SigmaAldrich, unless otherwise indicated.

Growth curves and cell growth. HepG2 and MHCC97H cells treated with different doses of DAPT were seeded onto 6-well cell culture plates at a density of $5 \times 10^{3}$ cells/well and were grown for up to 4 days. Each day, we used a hemocytometer to determine the number of cells. We used the relative density of the cells (vs. the density of the primary cells at 100\%) to establish the growth curve. Each experiment included six replications and was repeated three times. The data are summarized as means \pm SDs.

MTT assay. The HepG2 and MHCC97H cells treated with different doses of DAPT were seeded into 6-well cell culture plates at a density of $1 \times 10^{4}$ cells/well and were grown for up to 4 days. Cell viability was assessed using the 3-[4,5-dimethyl2-thiazolyl]-2,5-diphenyl-2H-tetrazolium bromide (MTT) assay (Sigma Chemicals Co.) in accordance with the manufacturer's protocol. Each experiment included six replications and was repeated three times. The data are summarized as means \pm SDs.

Small interfering RNA transfection. According to the protocol of Lipofectamine 2000, the HepG2 and MHCC97H cells were transfected with MMP-2 siRNA, MMP-9 siRNA, VEGF siRNA and siRNA control respectively. Cells transfected with siRNA were seeded into 6-well cell culture plates at a density of $1 \times 10^{5}$ cells/well. The cells were allowed to grow further for $24 \mathrm{~h}$ and were then harvested for further analysis.

Real-time reverse transcription-PCR analysis for gene expression. Total RNA from different cells was isolated by TRIzol (Invitrogen, Carlsbad, CA, USA) and purified using an RNeasy Mini kit and RNase-free DNase Set (Qiagen, Valencia, CA) according to the protocols of the manufacturer. Total RNA $(1 \mu \mathrm{g})$ from each sample was subjected to first-strand cDNA synthesis using TaqMan reverse transcription reagent kit (Applied Biosystems, Foster City, CA) in a total volume of $50 \mu \mathrm{l}$, including 6.25 units MultiScribe reverse transcriptase and 25 pmol random hexamers. The reverse transcription reaction was performed at $25^{\circ} \mathrm{C}$ for 10 min followed by $48^{\circ} \mathrm{C}$ for $30 \mathrm{~min}$ and $95^{\circ} \mathrm{C}$ for $5 \mathrm{~min}$. The primers used in the PCR reaction are as follows: Hes1, forward primer (5'-AGGCGGA CATTCTGGAAATG-3') and reverse primer (5'-TCGTTCA TGCACTCGCTGA-3'); Hes5, forward primer (5'-ACCGCAT CAACAGCAGCATT-3') and reverse primer (5'-AGGCTTT GCTGTGCTTCAGGT-3'); Hey1, forward primer (5'-AACTG TTGGTGGCCTGAATC-3') and reverse primer (5'-GCGGT AAATGCAGGCGTAT-3'); GAPDH, forward primer (5'-AA ATCCCATCACCATCTTCC-3') and reverse primer (5'-TCA CACCCATGACGAACA-3'). The primers were checked by running a virtual PCR and a primer concentration was optimized to avoid primer dimer formation. Also, dissociation curves were checked in order to avoid a non-specific amplification. Real-time PCR amplifications were undertaken in a Mx4000 Multiplex QPCR System (Stratagene, La Jolla, CA) using 2X SYBR-Green PCR Master mix (Applied Biosystems). One microliter of reverse transcription reaction was used for a total volume of $25 \mu \mathrm{l}$ quantitative PCR reactions. The thermal profile for SYBR real-time PCR was $95^{\circ} \mathrm{C}$ for $10 \mathrm{~min}$ followed by 40 cycles of $95^{\circ} \mathrm{C}$ for $15 \mathrm{sec}$ and $60^{\circ} \mathrm{C}$ for $1 \mathrm{~min}$. Data were analyzed according to the comparative $\mathrm{Ct}$ method and were normalized by GAPDH expression in each sample.

Protein extraction and western blotting. The cells were lysed in lysis buffer [50 mmol/l Tris (pH 7.5), $100 \mathrm{mmol} / \mathrm{l} \mathrm{NaCl}$, $1 \mathrm{mmol} / 1$ EDTA, $0.5 \% \mathrm{NP} 40,0.5 \%$ Triton X-100, $2.5 \mathrm{mmol} / \mathrm{l}$ sodium orthovanadate, $10 \mu \mathrm{l} / \mathrm{ml}$ protease inhibitor cocktail, and $1 \mathrm{mmol} / 1 \mathrm{PMSF}$ ] by incubating for $20 \mathrm{~min}$ at $4^{\circ} \mathrm{C}$. The protein concentration was determined using the Bio-Rad assay system (Bio-Rad Laboratories, Hercules, CA). Total proteins were fractionated using SDS-PAGE and were transferred onto nitrocellulose membranes. The membranes were blocked with $5 \%$ non-fat dried milk or bovine serum albumin in $1 \mathrm{X}$ TBS buffer containing $0.1 \%$ Tween-20 and then were incubated with the appropriate primary antibodies. Horseradish peroxidase-conjugated anti-rabbit or anti-goat IgG was used as the secondary antibody, and the protein bands were detected using the enhanced chemiluminescence detection system (Amersham Pharmacia Biotech, Amersham, UK). Quantification of western blots was performed using laser densitometry, and relative protein expression was then normalized to GAPDH levels in each sample. The results are 
A

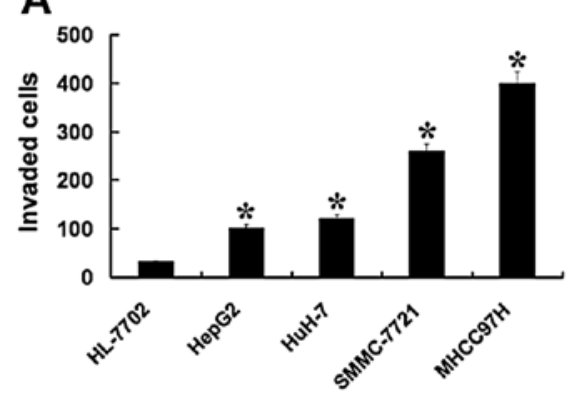

C

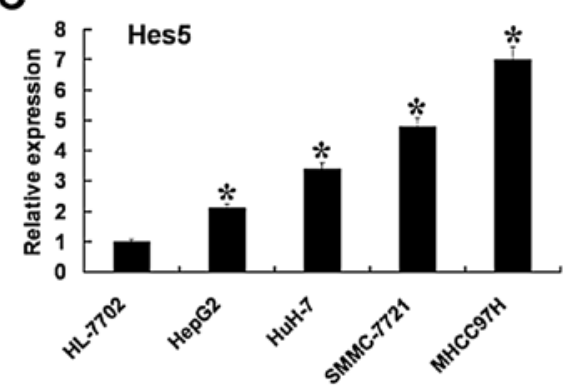

B

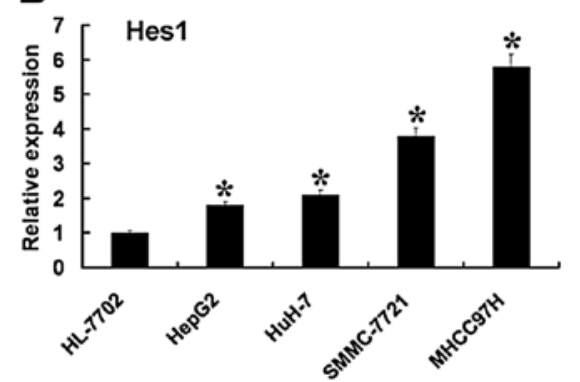

D

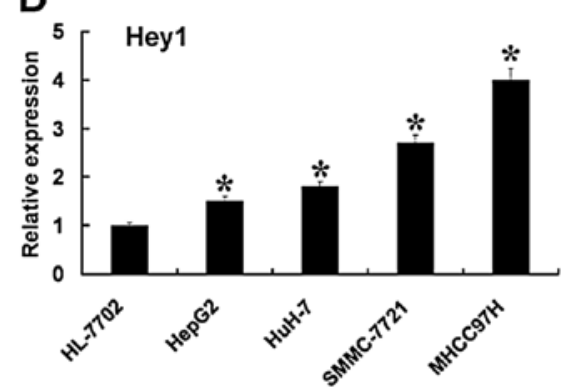

E

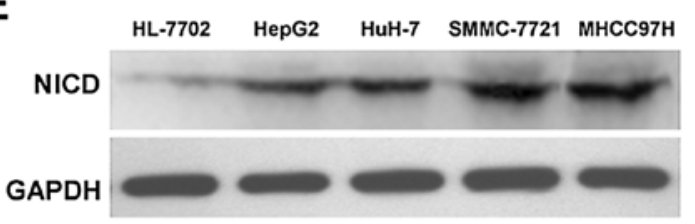

Figure 1. Expression of Notch signaling pathway in the liver non-tumor cell and HCC cells. (A) Using Transwell cell culture chambers, we detected the invasion capabilities of liver non-tumor cell and different HCC cell lines. (B-E) RT-PCR and western blotting analyzed the expression of the Notch signaling pathway in liver non-tumor and HCC cells. The expression of the Notch signaling pathway was normalized to GAPDH levels (Hes1 or Hes5 or Hey1 or NICD/ GAPDH). The data represent mean $\pm \mathrm{SD},{ }^{*} \mathrm{P}<0.05$ compared with the HL-7702 cells.

presented as the means of three independent experiments with error bars representing SDs. For reprobing, membranes were incubated for $30 \mathrm{~min}$ at $50^{\circ} \mathrm{C}$ in a buffer containing $2 \% \mathrm{SDS}$, $62.5 \mathrm{mmol} / \mathrm{l}$ Tris (pH 6.7), and $100 \mathrm{mmol} / \mathrm{l} 2$-mercaptoethanol, washed and incubated with the desired primary antibody.

Invasion assays. The cell invasion capacity was analyzed by using Matrigel-coated Transwell cell culture chambers (8 $\mu \mathrm{m}$ pore size) (Millipore, Billerica, MA, USA). Briefly, treated cells $\left(5 \times 10^{4}\right.$ cells/well) were serum-starved for $24 \mathrm{~h}$ and were plated in the upper insert of a 24 -well culture plates in serum-free medium. Medium containing $10 \%$ serum as a chemoattractant was added to the well. The cells were incubated under normoxic conditions for $24 \mathrm{~h}$. Non-invading cells were removed from the upper surface by scrubbing with a cotton swab, after which the membrane was fixed with $4 \%$ formaldehyde for $10 \mathrm{~min}$ at room temperature and was stained with $0.5 \%$ crystal violet for $10 \mathrm{~min}$. Finally, invasive cells were counted at x200 magnification from 10 different fields of each filter. For treatment with DAPT and PD98059, the cells were pretreated for $2-4 \mathrm{~h}$, and the treatment continued during the invasion experiment.

ELISA assay. Enzyme-linked immunosorbent assay (ELISA) technique (Amersham Pharmacia Biotech) was used to quantify the activity of individual MMP-2, MMP-9, VEGF and ERK1/2. The samples were thawed on ice, and all reagents were equilibrated to room temperature. Assays were carried out according to the manufacturer's instructions.

Statistical analysis. Each experiment was repeated at least three times. All data were summarized and are presented as means \pm SDs. The differences among means were statistically analyzed using a t-test. All statistical analyses were performed using the SPSS 13.0 software (SPSS Inc., Chicago, IL, USA). $\mathrm{P}<0.05$ was considered as statistically significant.

\section{Results}

Expression of Notch signaling pathway increased in HCC cells. As illustrated in Fig. 1A, the HCC cell lines showed higher levels of penetration through Transwell cell culture chambers, with Matrigel-coating vs. the liver non-tumor cell. In HCC cell lines, the level of penetration was the lowest in HepG2 cells and was the highest in MHCC97H cells. These results demonstrated that HCC cells had higher invasion capabilities than the liver non-tumor cells. In HCC cells, HepG2 cells had the lowest invasion capability and the $\mathrm{MHCC} 97 \mathrm{H}$ cells had the highest invasion capability.

Next, we examined the expression of the Notch signaling pathway in the liver non-tumor and HCC cells. RT-PCR analysis showed that HCC cells had higher mRNA levels of the Notch signaling pathway downstream target genes Hes1, Hes5, and Heyl compared to the liver non-tumor cells (Fig. 1B-D). 
A

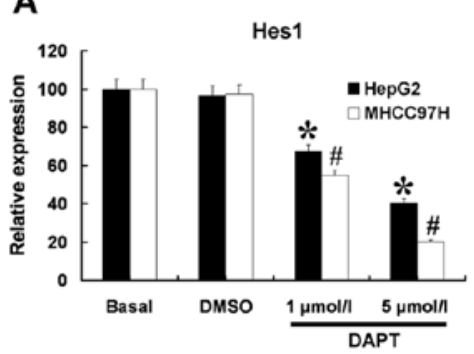

B

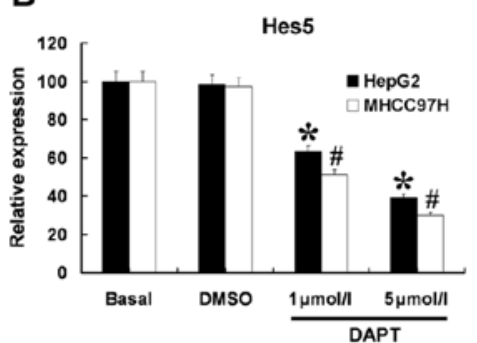

C

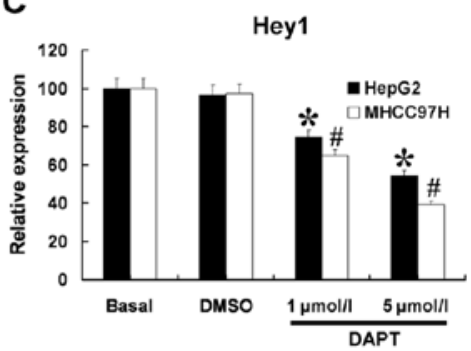

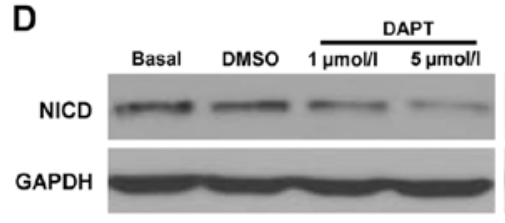
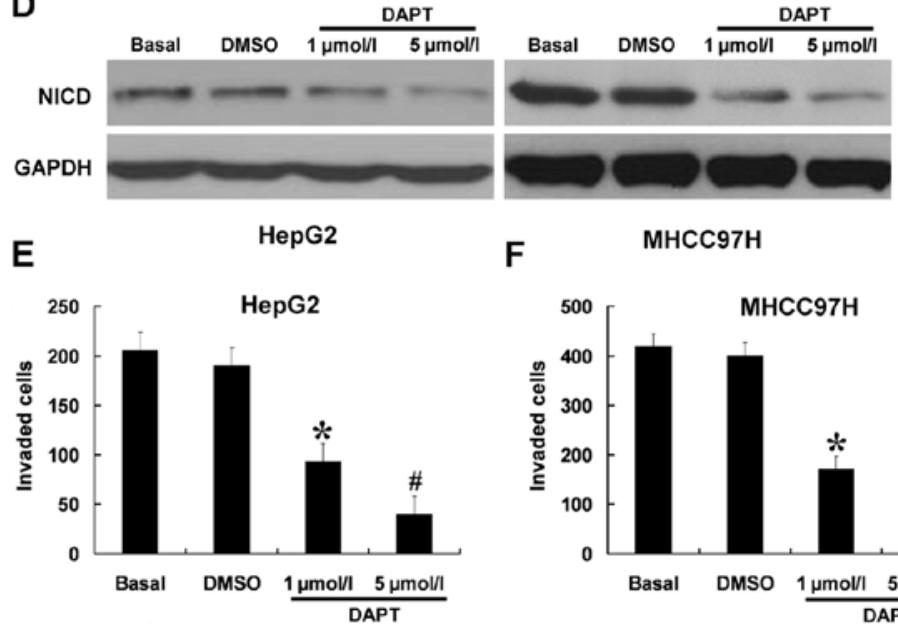

$\mathbf{F}$

MHCC97H

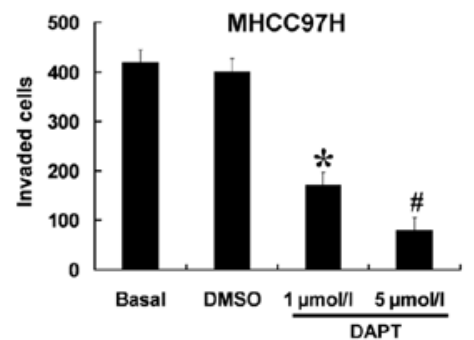

Figure 2. The effects of DAPT on the expression of Notch signaling pathway and the invasion capacities of HCC cells. HepG2 and MHCC97H cells were treated with DAPT at different doses (1 and $5 \mu \mathrm{mol} / \mathrm{l})$. The non-treated (basal cells) or treated with DMSO cells served as controls. (A-C) Expression of Hes1, Hes5 and Hey1 at the mRNA level were measured by RT-PCR. (D) The expression of NICD at the protein level was measured by western blotting. (E and F) The invasion capacities of the treated HepG2 and MHCC97H cells were measured by using Transwell cell culture chambers. The data represent mean \pm SD, ${ }^{*} \mathrm{P}<0.05$ compared with HepG2 cells treated with DMSO, ${ }^{\text {} P} \mathrm{P}<0.05$ compared with MHCC $97 \mathrm{H}$ cells treated with DMSO.

A
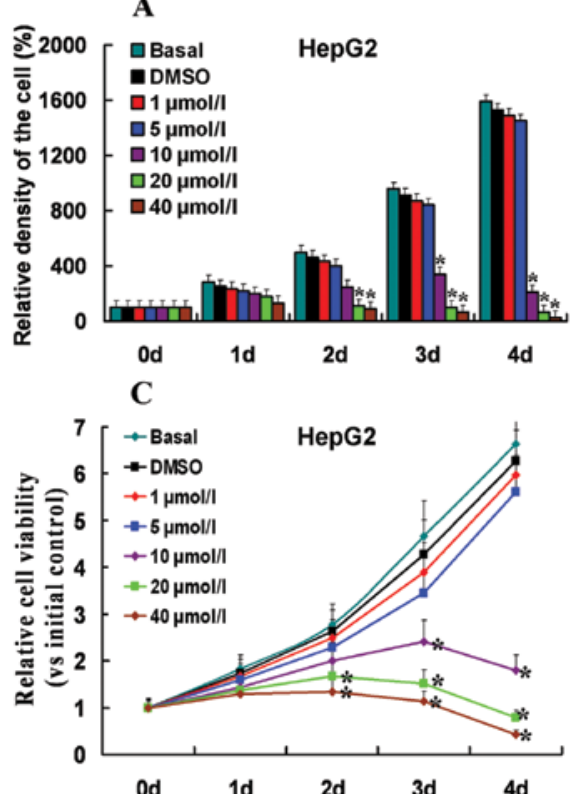

B

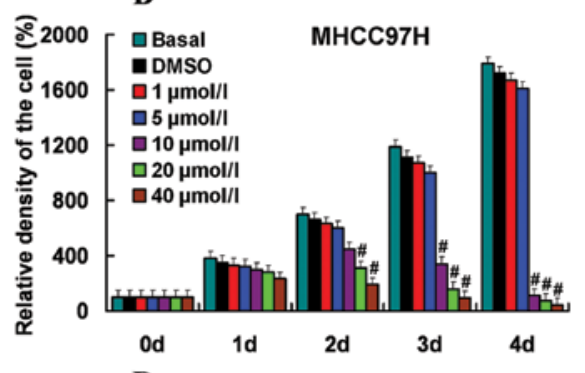

D

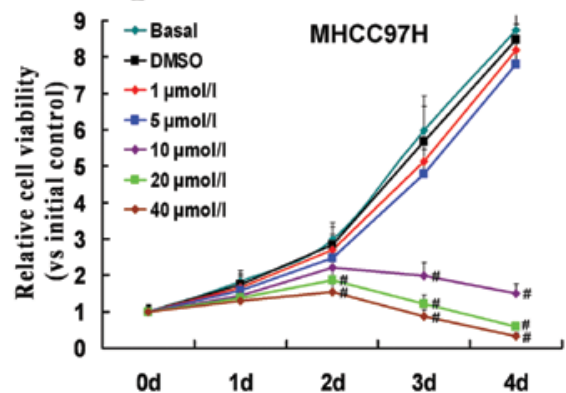

Figure 3. Effects of DAPT on the growth and viability of HepG2 and MHCC97H cells. The HepG2 and MHCC97H cells were treated with different doses of DAPT $(1,5,10,20$ and $40 \mu \mathrm{mol} / \mathrm{l})$ for 4 days. The non-treated (basal) cells or the cells treated with DMSO served as controls. (A and B) Density of HepG2 and MHCC 97H cells were measured using a hemocytometer. (C and D) Viability of HepG2 and MHCC97H cells measured by the MTT aasay. The data represent means \pm SDs, ${ }^{*} \mathrm{P}<0.05$ compared to HepG2 cells treated with DMSO, ${ }^{*} \mathrm{P}<0.05$ compared to MHCC $97 \mathrm{H}$ cells treated with DMSO.

NICD also exhibited similar increased tendencies regarding the protein levels (Fig. 1E). These results may illustrate that the expression of the Notch signaling pathway is upregulated in HCC cells. The expression of Hes1, Hes5, Heyl and NICD 

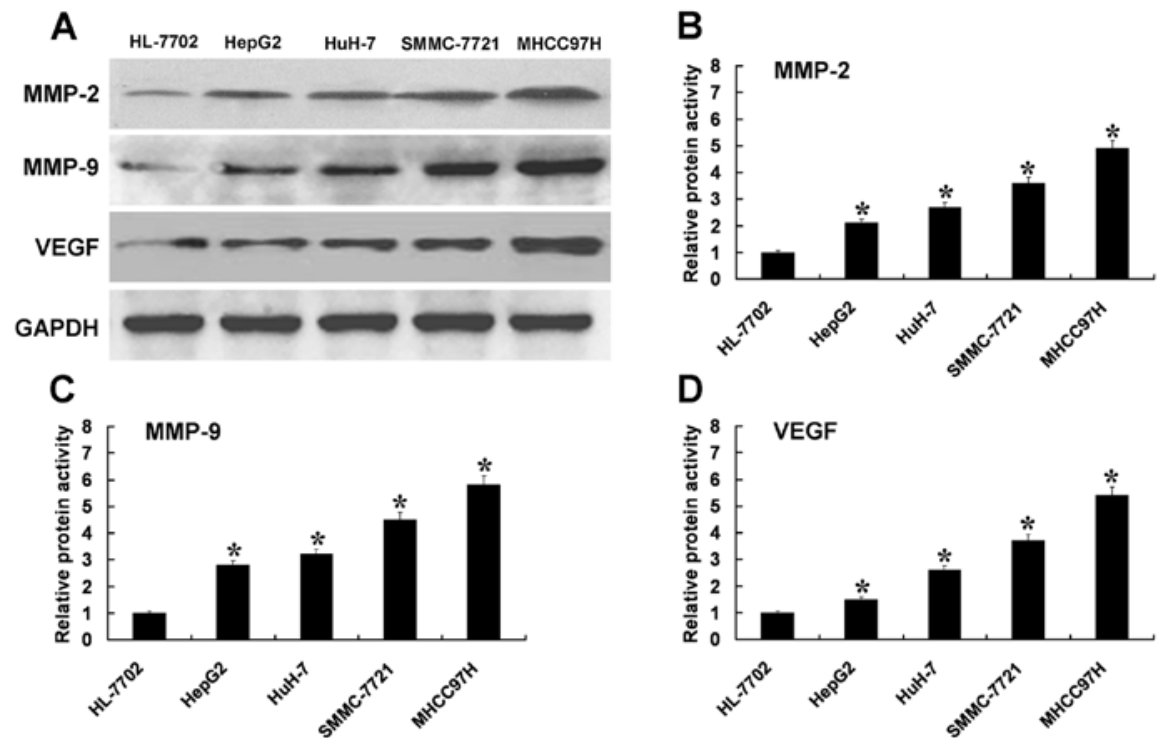

D

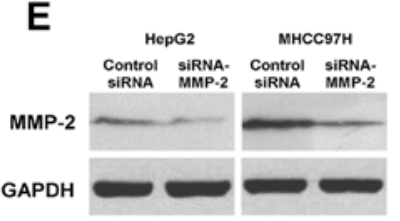

H
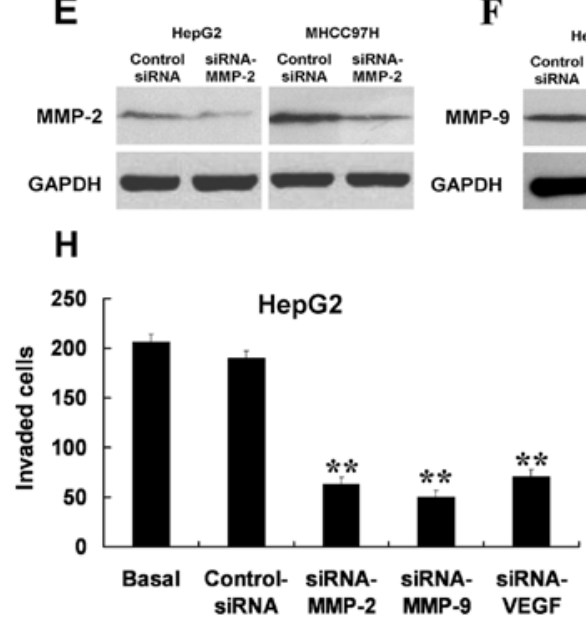

$\mathbf{F}$

epG2

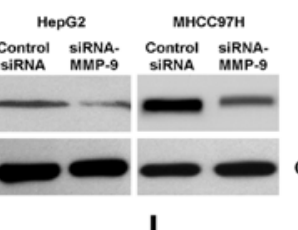

I

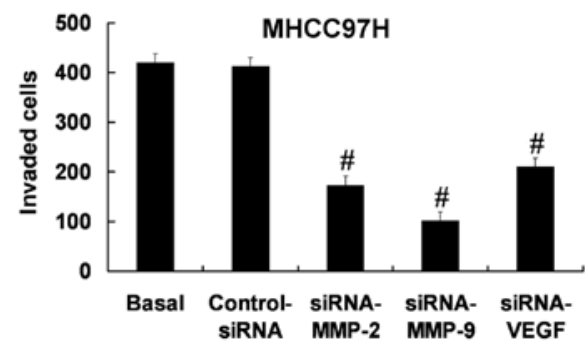

Figure 4. Downregulation of MMP-2, MMP-9 and VEGF decreased HCC cell invasion. (A) Protein expression of MMP-2, MMP-9 and VEGF were measured by western blotting in the liver non-tumor and HCC cells. (B-D) The proteolytic activity of MMP-2, MMP-9 and VEGF were measured in the liver non-tumor cell and HCC cells by the ELISA assay. (E-G) HepG2 and MHCC97H cells treated with siRNA transfection showed lower protein expression of MMP-2, MMP-9 and VEGF as confirmed by western blotting. (H-I) Using Transwell cell culture chambers, the MMP-2, MMP-9 or VEGF siRNA-transfected cells showed lower penetration through the Matrigel-coated membrane compared with control siRNA-transfected cells. The data represent mean $\pm \mathrm{SD},{ }^{*} \mathrm{P}<0.05$ compared with HL-7702; ${ }^{* *} \mathrm{P}<0.05$ compared to the HepG2 cell treated with control siRNA; ${ }^{*} \mathrm{P}<0.05$ compared to the MHCC $97 \mathrm{H}$ cell treated with control siRNA.

were the lowest in HepG2 cells and were the highest in MHCC 97H cells. This indicates that the upregulated levels of the Notch signaling pathway may have a correlation with the invasion capability.

DAPT can efficiently downregulate the Notch signaling pathway and inhibit invasion in HCC cells. Invasion capacity was the lowest in the HepG2 cell and the highest in the MHCC97H cells. Therefore, we only used HepG2 and MHCC 97H cells for the next experiment. We further investigated the role of DAPT on the expression of the Notch signaling pathway in HepG2 and MHCC97H cells. The cells were treated with DAPT at different doses $(1$ and $5 \mu \mathrm{mol} / \mathrm{l})$ and the cells not-treated or treated with DMSO were used as controls. The mRNA expression levels of Hes1, Hes5, and Hey1 were measured by RT-PCR and protein expression of NICD was measured by western blotting. As shown in Fig. 2A-D, HepG2 and MHCC97H cells treated with DAPT reduced the mRNA expression levels of Hes1, Hes5, Hey1, and the protein expression of NICD in a dose-dependent manner. We next sought to determine whether DAPT affected the invasion capabilities of HepG2 and MHCC97H cells. As shown in Fig. 2E and F, the invasion capabilities of $\mathrm{HepG} 2$ and $\mathrm{MHCC} 97 \mathrm{H}$ cells were strongly inhibited by DAPT in a dose-dependent manner. As shown in Fig. 3, the indicated concentrations of DAPT (1 or $5 \mu \mathrm{mol} / \mathrm{l}$ ) had no effect on the growth and viability of HepG2 and MHCC $97 \mathrm{H}$ cells. These results indicate that the inhibitory effects of DAPT ( 1 or $5 \mu \mathrm{mol} / \mathrm{l})$ on cell invasion were independent of cellular cytotoxicity.

MMP-2, MMP-9 and VEGF may participate in HCC cell invasion. MMP-2, MMP-9 and VEGF are associated with enhanced invasion of tumor cells. With western blotting, the protein expressions of MMP-2, MMP-9 and VEGF were upregulated in the HCC cells compared with the liver nontumor cells at the protein level (Fig. 4A). The proteolytic 


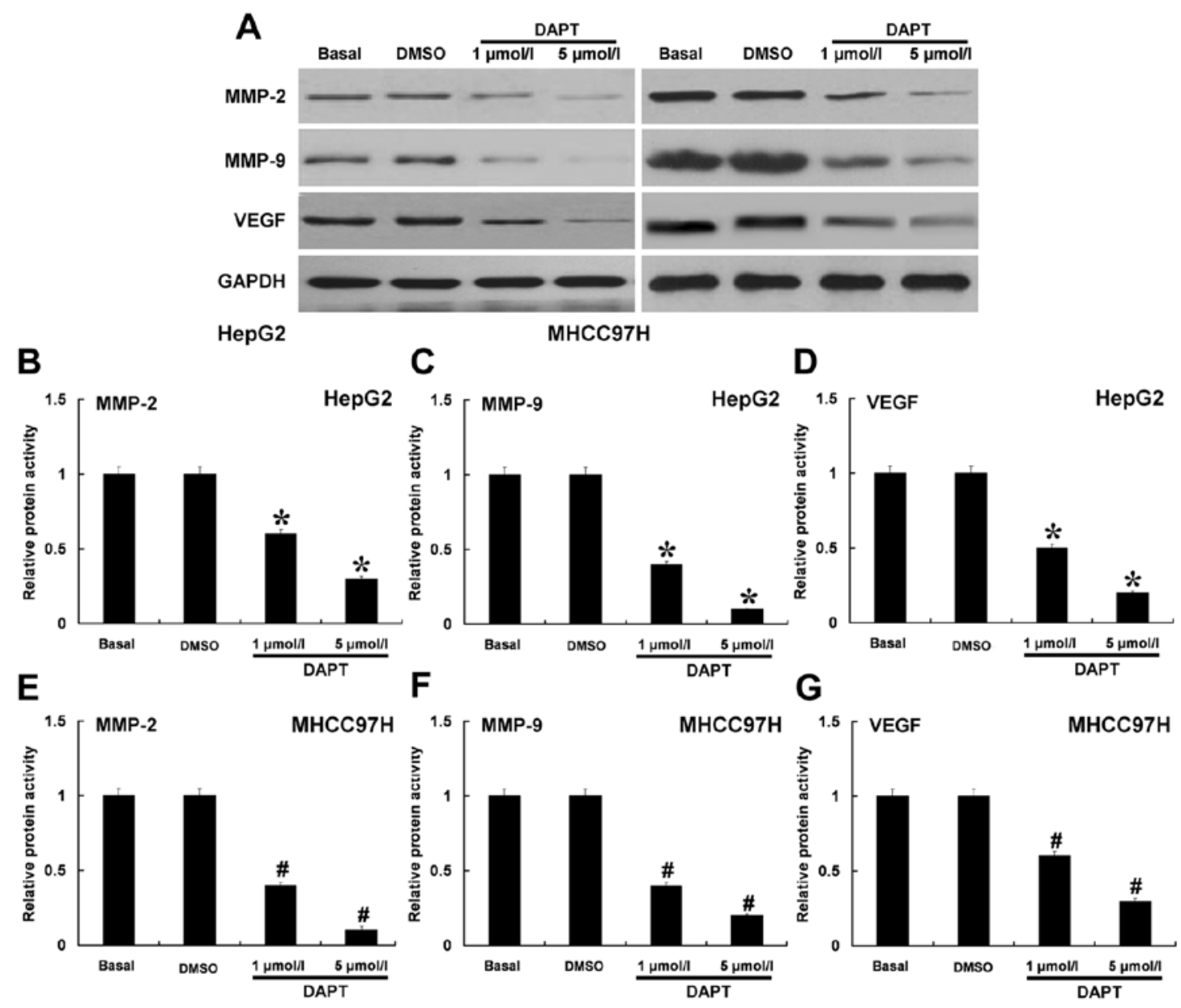

Figure 5. In HepG2 and MHCC97H cells, the protein expression levels and proteolytic activities of MMP-2, MMP-9 or VEGF were downregulated by DAPT in a dose-dependent manner. The HepG2 and MHCC97H cells were treated with DAPT at different doses ( 1 and $5 \mu \mathrm{mol} / \mathrm{l})$. The non-treated (basal) cells or those treated with DMSO served as control. (A) Protein expression levels of MMP-2, MMP-9 or VEGF were measured by western blotting in HepG2 and MHCC97H cells. (B-G) The proteolytic activities of MMP-2, MMP-9 or VEGF were measured by ELISA assay. "P<0.05 compared to HepG2 cells treated with DMSO, ${ }^{\prime \prime} \mathrm{P}<0.05$ compared to MHCC $97 \mathrm{H}$ cells treated with DMSO.

activities of MMP-2, MMP-9 and VEGF exhibited similar increased tendencies in HCC cells (Fig. 4B-D). The protein expression levels and proteolytic activities of MMP-2, MMP-9 and VEGF were the lowest in the HepG2 cells and the highest in the MHCC $97 \mathrm{H}$ cells. These results also showed upregulated levels of MMP-2, MMP-9 and VEGF may correlate with invasion capability. The HepG2 and MHCC $97 \mathrm{H}$ cells were transfected with human MMP-2 siRNA, MMP-9 siRNA, and VEGF siRNA. The cells non-transfected and transfected with control siRNA were as control. siRNA can efficiently downregulate the expression of MMP-2, MMP-9 and VEGF (Fig. 4E-G). Cells transfected with MMP-2 siRNA or MMP-9 siRNA or VEGF siRNA showed a lower level of penetration through the membrane, compared with control siRNA-transfected cells (Fig. 4H and I). These results indicated that MMP-2, MMP-9 and VEGF may participate in HCC cell invasion.

Inhibition of Notch signaling pathway by DAPT decreased the protein expression and proteolytic activities of MMP-2, $M M P-9$ or VEGF. We determined whether inhibition of Notch signaling pathway by different doses of DAPT could have effects on MMP-2, MMP-9 and VEGF. Western blotting and
ELISA assay were used to analyze the protein expression and proteolytic activity. DAPT was able to effectively inhibit the protein expressions of MMP-2, MMP-9 and VEGF in a dosedependent manner in HepG2 and MHCC97H cells (Fig. 5A). Using ELISA assay (Fig. 5B-G), we found that DAPT also could effectively inhibit the proteolytic activities of MMP-2, MMP-9 and VEGF. These results indicated that the Notch signaling pathway may regulate MMP-2, MMP-9 and VEGF in HCC cells.

Downregulation of the Notch signaling pathway by DAPT inhibited ERK1/2 activity resulting in inhibition of the invasion of HCC cells. ERK1/2 is known to play a major role in signaling pathways concerning invasion, and regulates the expression of MMPs and VEGF. We investigated whether the antagonistic effects of DAPT on the upregulation of MMP-2, MMP-9 and VEGF expression and decreased invasion in HCC cells could be attributed to the inhibition of ERK1/2. As shown in Fig. 6A, using western blot analysis, we found that increasing doses of DAPT abolished ERK1/2 phosphorylation in a dose-dependent manner. We further confirmed that DAPT inhibited ERK1/2 activity by ELISA assay (Fig. 6B and C). The results indicated that DAPT significantly inhibited the activity 

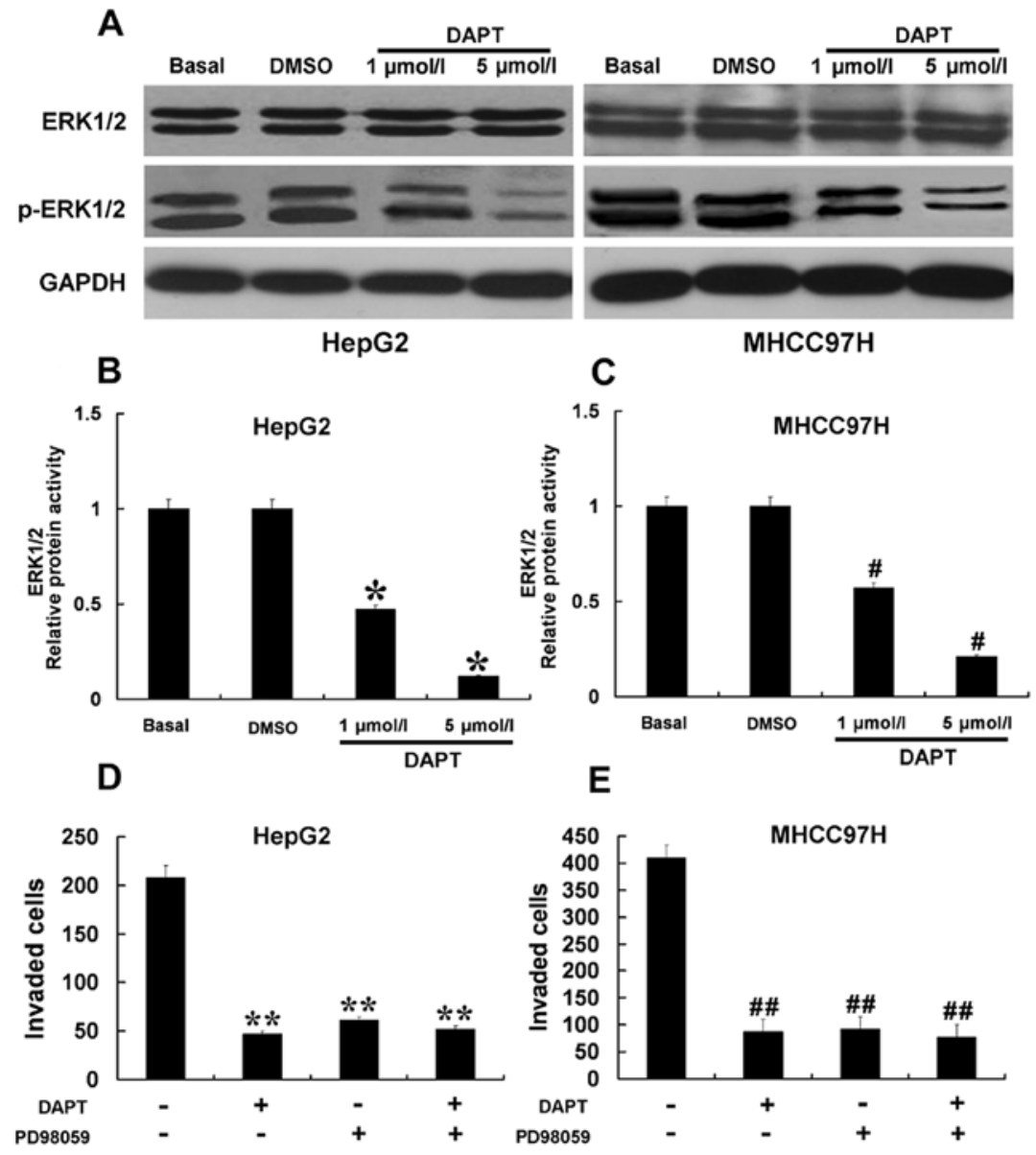

Figure 6. DAPT inhibits ERK1/2 activity resulting in the inhibition of invasion of HCC cells. The HepG2 and MHCC97H cells were treated with DAPT at different doses (1 and $5 \mu \mathrm{mol} / 1)$. Non-treated (basal) cells or cells treated with DMSO served as controls. (A) ERK1/2 phosphorylation was measured by western blotting in different treated HepG2 and MHCC97H cells. (B and C) The same treated HCC cells were subjected to ELISA assay for analysis of ERK1/2 activity. (D and E) HepG2 and MHCC97H cells were treated with $5 \mu \mathrm{mol} / 1 \mathrm{DAPT}$ and/or $10 \mu \mathrm{mol} / 1 \mathrm{PD} 98059$ to evaluate invasion capacities. ${ }^{*} \mathrm{P}<0.05$ compared to HepG2 cell treated with DMSO, ${ }^{\text {"P }}<0.05$ compared to MHCC97H cell treated with DMSO. ${ }^{* *} \mathrm{P}<0.05$ compared to non-treated HepG2 cells, ${ }^{\# \#} \mathrm{P}<0.05$ compared to the non-treated MHCC97H cells.

of ERK1/2 in HCC cells. To further study the relationship between ERK1/2 activity and the Notch signaling pathway in the control of tumor invasion, HepG2 and MHCC97H cells were treated with $10 \mu \mathrm{mol} / 1 \mathrm{PD} 98059$ and $5 \mu \mathrm{mol} / \mathrm{l}$ DAPT to block ERK1/2 activity and the Notch signaling pathway, respectively (Fig. 6D and E). Treatment with PD98059 or DAPT alone reduced HepG2 and MHCC97H cell invasion. However, treatment with PD98059 in combination with DAPT did not block these biological functions of HCC cells to a greater extent than treatment with PD98059 or DAPT alone. These results suggest that the Notch signaling pathway may modulate HCC cell invasion through ERK1/2 regulating MMP-2, MMP-9 and VEGF.

\section{Discussion}

The high recurrence rate of intrahepatic and distant metastasis is a major obstacle in improving the survival rate of patients with HCC (20). If the mechanisms regulating HCC invasion can be clearly defined, there are likely to be key elements that can be exploited therapeutically, reducing metastasis and improving survival. There has been increasing evidence indicating that the Notch signaling pathway increases the metastatic potential of tumor cells by increasing processes such as invasion $(21,22)$. Our present studies investigated the role of the Notch signaling pathway on HCC cell invasion. Our findings indicate that the inhibition of the Notch signaling pathway decreased the protein expression and proteolytic activities of MMP-2, MMP-9, and VEGF by inhibiting ERK1/2 activity and suppressed HCC cell invasion. Taken together, the data indicate that inhibition of the Notch signaling pathway should be further evaluated in $\mathrm{HCC}$ invasion therapy.

Invasion and metastasis are the processes by which tumor spreads from the place it first appears as a primary tumor to distant locations in the body. This includes a series of sequential steps, including tumor-induced angiogenesis, tumor invasion, and the establishment of metastatic foci at the secondary site involving various molecules $(23,24)$. The MMPs family proteins are the proteolytic enzymes in ECM that contribute to tumor invasion, angiogenesis and metastasis (25). Among the previously reported human MMPs, MMP-2 and MMP-9 have been implicated in invasion and metastasis because of their role in the degradation of basement membrane collagen $(26,27)$. It was reported that MMP-2 and MMP-9 are correlated with an aggressive, invasive or metastatic tumor phenotype $(28,29)$. It is well known that the MMP inhibitors 
block endothelial cell activities which are essential for new vessel development leading to proliferation and invasion (30). Therefore, MMP-2 and MMP-9 are considered therapeutic targets of anticancer drugs based on the degrading action of both enzymes on gelatins which are major components of the basement membrane. Another important molecule involved in tumor cell invasion and metastasis is VEGF. The expression of VEGF is commonly found to be upregulated in tumors and there was a trend toward an association between the expression of VEGF and distant metastasis. Investigations by other laboratories have shown that VEGF promotes migration and invasion of tumor cells $(31,32)$. Here, we showed that the protein expressions and proteolytic activities of MMP-2, MMP-9 and VEGF were higher in HCC cells. The inhibition of MMP-2, MMP-9 and VEGF by siRNA can also decrease the invasion capabilities of HCC cells. These results indicate that MMP-2, MMP-9 and VEGF may participate in HCC cell invasion.

The Notch signaling pathway is involved in the carcinogenesis, progress, invasion and neurovascular formation of many malignant tumors (22,33-35). The Notch signaling pathway can regulate MMP-2, MMP-9 and VEGF (22,36-40), which are important in the processes of invasion and metastasis of tumor. In the current study, the invasion capabilities of HepG2 and MHCC97H cells treated with DAPT decreased. We showed that an increase in the DAPT dose in response to Notch signaling pathway inhibition resulted in suppression of MMP-2, MMP-9 and VEGF. These results suggest that the inhibitory effect of DAPT on HCC cell invasion can be partially attributable to the downregulation of MMP-2, MMP-9 and VEGF. Some studies have shown that the Notch signaling pathway regulates MMPs and VEGF partly due to activation of the NF- $\gamma \mathrm{B}$ pathway in cell invasion in pancreatic cancer cells (22). However, the potential mechanisms between Notch signaling pathway, MMPs and VEGF in HCC invasion are poorly understood.

Extracellular signal-regulated kinase 1 and 2 (ERK1/2) belongs to the family of mitogen-activated protein kinases (MAPKs) which play a major role in the signaling pathways concerning scattering/motility, invasion, proliferation and survival (41-43). ERK1/2 activation has also been reported to regulate the expression of a variety of important genes in some cellular responses, including metastasis related genes, such as VEGF and MMP-2 and -9 (44-47). Because ERK1/2 plays an important role in many cellular processes, studies on the interaction of ERK1/2 activation with other cell signal transduction pathways, including the Notch signaling pathway, has received increased attention in recent years. The Notch signaling pathway has also been reported to crosstalk with the ERK1/2 pathway (48). In the present study, we showed that the downregulation of the Notch signaling pathway by DAPT reduced ERK1/2 activity and concomitantly inhibited the protein expression and proteolytic activities of MMP-2, MMP-9 and VEGF. We also found that the inhibited Notch signaling pathway and/ or ERK1/2 has the same role in suppressing invasion of HCC cells. Thus, the downregulation of the Notch signaling pathway results in lower ERK1/2 activity and its downstream targets (MMP-2 and -9 and VEGF). Therefore, it is possible that Notch signaling pathway induced HCC cell invasion is partly due to the activation of the ERK1/2 pathway.
Taken together, our data showed that the Notch signaling pathway inhibitor could suppress invasion of HCC cells via ERK1/2 signaling pathways, resulting in the downregulation of MMP-2, MMP-9 and VEGF. Inhibition of Notch signaling pathway could be useful as a therapeutic target for inhibiting HCC invasion. Further studies will elucidate the mechanism of the Notch signaling pathway and ERK1/2 interaction.

\section{Acknowledgements}

We are grateful to Fuqin Zhang who provided technical support. This study was supported by grants from the National Natural Science Foundation of China (grants no. 30872480) and the Major Program of the National Natural Science Foundation of China (grants no. 81030010/H0318).

\section{References}

1. Yang JD, Nakamura I and Roberts LR: The tumor microenvironment in hepatocellular carcinoma: current status and therapeutic targets. Semin Cancer Biol 21: 35-43, 2011.

2. Thomas MB and Zhu AX: Hepatocellular carcinoma: the need for progress. J Clin Oncol 23: 2892-2899, 2005.

3. Tang DJ, Dong SS, Ma NF, et al: Overexpression of eukaryotic initiation factor 5A2 enhances cell motility and promotes tumor metastasis in hepatocellular carcinoma. Hepatology 51: 1255-1263, 2010.

4. Itoh Y and Nagase H: Matrix metalloproteinases in cancer. Essays Biochem 38: 21-36, 2002.

5. Zeng ZS, Cohen AM and Guillem JG: Loss of basement membrane type IV collagen is associated with increased expression of metalloproteinases 2 and 9 (MMP-2 and MMP-9) during human colorectal tumorigenesis. Carcinogenesis 20: 749-755, 1999.

6. Komatsu K, Nakanishi Y, Nemoto N, Hori T, Sawada T and Kobayashi M: Expression and quantitative analysis of matrix metalloproteinase-2 and -9 in human gliomas. Brain Tumor Pathol 21: 105-112, 2004.

7. Folkman J: What is the evidence that tumors are angiogenesis dependent? J Natl Cancer Inst 82: 4-6, 1990.

8. Joo YE, Sohn YH, Lee WS, et al: Expression of vascular endothelial growth factor and p53 in pancreatic carcinomas. Korean J Intern Med 17: 153-159, 2002.

9. Zeng H, Datta K, Neid M, Li J, Parangi S and Mukhopadhyay D: Requirement of different signaling pathways mediated by insulin-like growth factor-I receptor for proliferation, invasion, and VPF/VEGF expression in a pancreatic carcinoma cell line. Biochem Biophys Res Commun 302: 46-55, 2003.

10. Artavanis-Tsakonas S, Rand MD and Lake RJ: Notch signaling: cell fate control and signal integration in development. Science 284: 770-776, 1999.

11. Miele L and Osborne B: Arbiter of differentiation and death: Notch signaling meets apoptosis. J Cell Physiol 181: 393-409, 1999.

12. Allenspach EJ, Maillard I, Aster JC and Pear WS: Notch signaling in cancer. Cancer Biol Ther 1: 466-476, 2002.

13. Nickoloff BJ, Osborne BA and Miele L: Notch signaling as a therapeutic target in cancer: a new approach to the development of cell fate modifying agents. Oncogene 22: 6598-6608, 2003.

14. Leong KG and Karsan A: Recent insights into the role of Notch signaling in tumorigenesis. Blood 107: 2223-2233, 2006.

15. Radtke F and Raj K: The role of Notch in tumorigenesis: oncogene or tumour suppressor? Nat Rev Cancer 3: 756-767, 2003.

16. Zweidler-McKay PA, He Y, Xu L, et al: Notch signaling is a potent inducer of growth arrest and apoptosis in a wide range of B-cell malignancies. Blood 106: 3898-3906, 2005.

17. Kunnimalaiyaan $\mathrm{M}$ and Chen $\mathrm{H}$ : Tumor suppressor role of Notch-1 signaling in neuroendocrine tumors. Oncologist 12: 535-542, 2007.

18. Proweller A, Tu L, Lepore JJ, et al: Impaired notch signaling promotes de novo squamous cell carcinoma formation. Cancer Res 66: 7438-7444, 2006. 
19. Seiffert D, Bradley JD, Rominger CM, et al: Presenilin-1 and -2 are molecular targets for gamma-secretase inhibitors. J Biol Chem 275: 34086-34091, 2000.

20. Tung-Ping Poon R, Fan ST and Wong J: Risk factors, prevention, and management of postoperative recurrence after resection of hepatocellular carcinoma. Ann Surg 232: 10-24, 2000.

21. Sahlgren C, Gustafsson MV, Jin S, Poellinger L and Lendahl U: Notch signaling mediates hypoxia-induced tumor cell migration and invasion. Proc Natl Acad Sci USA 105: 6392-6397, 2008.

22. Wang Z, Banerjee S, Li Y, Rahman KM, Zhang Y and Sarkar FH: Down-regulation of notch-1 inhibits invasion by inactivation of nuclear factor-kappaB, vascular endothelial growth factor, and matrix metalloproteinase-9 in pancreatic cancer cells. Cancer Res 66: 2778-2784, 2006

23. Fidler IJ: The pathogenesis of cancer metastasis: the 'seed and soil' hypothesis revisited. Nat Rev Cancer 3: 453-458, 2003.

24. Weiss L: Metastasis of cancer: a conceptual history from antiquity to the 1990s. Cancer Metastasis Rev 19: 193-383, 2000.

25. Vihinen P and Kahari VM: Matrix metalloproteinases in cancer: prognostic markers and therapeutic targets. Int J Cancer 99: 157-166, 2002

26. Curran S and Murray GI: Matrix metalloproteinases: molecular aspects of their roles in tumour invasion and metastasis. Eur J Cancer 36: 1621-1630, 2000.

27. John A and Tuszynski G: The role of matrix metalloproteinases in tumor angiogenesis and tumor metastasis. Pathol Oncol Res 7: 14-23, 2001 .

28. Cockett MI, Murphy G, Birch ML, et al: Matrix metalloproteinases and metastatic cancer. Biochem Soc Symp 63: 295-313, 1998.

29. Bianco FJ Jr, Gervasi DC, Tiguert R, et al: Matrix metalloproteinase-9 expression in bladder washes from bladder cancer patients predicts pathological stage and grade. Clin Cancer Res 4: 3011-3016, 1998.

30. Murphy AN, Unsworth EJ and Stetler-Stevenson WG: Tissue inhibitor of metalloproteinases-2 inhibits bFGF-induced human microvascular endothelial cell proliferation. J Cell Physiol 157: 351-358, 1993

31. Wey JS, Fan F, Gray MJ, et al: Vascular endothelial growth factor receptor-1 promotes migration and invasion in pancreatic carcinoma cell lines. Cancer 104: 427-438, 2005.

32. Takahashi Y, Kitadai Y, Bucana CD, Cleary KR and Ellis LM: Expression of vascular endothelial growth factor and its receptor, $\mathrm{KDR}$, correlates with vascularity, metastasis, and proliferation of human colon cancer. Cancer Res 55: 3964-3968, 1995.

33. Balint K, Xiao M, Pinnix CC, et al: Activation of Notch1 signaling is required for beta-catenin-mediated human primary melanoma progression. J Clin Invest 115: 3166-3176, 2005.

34. Jundt F, Anagnostopoulos I, Forster R, Mathas S, Stein H and Dorken B: Activated Notch1 signaling promotes tumor cell proliferation and survival in Hodgkin and anaplastic large cell lymphoma. Blood 99: 3398-3403, 2002.
35. Buchler P, Gazdhar A, Schubert M, et al: The Notch signaling pathway is related to neurovascular progression of pancreatic cancer. Ann Surg 242: 791-801, 2005.

36. Yu B, Wei J, Qian X, Lei D, Ma Q and Liu Y: Notch1 signaling pathway participates in cancer invasion by regulating MMPs in lingual squamous cell carcinoma. Oncol Rep 27: 547-552, 2012.

37. Wang $\mathrm{J}, \mathrm{Fu} \mathrm{L}, \mathrm{Gu} F$ and Ma Y: Notch1 is involved in migration and invasion of human breast cancer cells. Oncol Rep 26: 1295-1303, 2011

38. Delbosc S, Glorian M, Le Port AS, Bereziat G, Andreani M and Limon I: The benefit of docosahexanoic acid on the migration of vascular smooth muscle cells is partially dependent on Notch regulation of MMP-2/-9. Am J Pathol 172: 1430-1440, 2008.

39. Xiong HQ, Abbruzzese JL, Lin E, Wang L, Zheng L and Xie K NF-kappaB activity blockade impairs the angiogenic potential of human pancreatic cancer cells. Int J Cancer 108: 181-188, 2004.

40. Funahashi Y,Shawber CJ, Sharma A,Kanamaru E, Choi YK and Kitajewski J: Notch modulates VEGF action in endothelial cells byinducing Matrix Metalloproteaseactivity. VascCell3:2,2011.

41. Chan-Hui PY and Weaver R: Human mitogen-activated protein kinase kinase kinase mediates the stress-induced activation of mitogen-activated protein kinase cascades. Biochem J 336: 599-609, 1998

42. Trusolino L and Comoglio PM: Scatter-factor and semaphorin receptors: cell signalling for invasive growth. Nat Rev Cancer 2: 289-300, 2002

43. Chen PN, Hsieh YS, Chiou HL and Chu SC: Silibinin inhibits cell invasion through inactivation of both PI3K-Akt and MAPK signaling pathways. Chem Biol Interact 156: 141-150, 2005.

44. Arai K, Lee SR and Lo EH: Essential role for ERK mitogenactivated protein kinase in matrix metalloproteinase-9 regulation in rat cortical astrocytes. Glia 43: 254-264, 2003.

45. Zeigler ME, Chi Y, Schmidt T and Varani J: Role of ERK and JNK pathways in regulating cell motility and matrix metalloproteinase 9 production in growth factor-stimulated human epidermal keratinocytes. J Cell Physiol 180: 271-284, 1999.

46. Giuliani N, Lunghi P, Morandi F, et al: Downmodulation of ERK protein kinase activity inhibits VEGF secretion by human myeloma cells and myeloma-induced angiogenesis. Leukemia 18: 628-635, 2004

47. Okajima E and Thorgeirsson UP: Different regulation of vascular endothelial growth factor expression by the ERK and p38 kinase pathways in v-ras, v-raf, and v-myc transformed cells. Biochem Biophys Res Commun 270: 108-111, 2000.

48. Wang Z, Li Y, Banerjee S and Sarkar FH: Exploitation of the Notch signaling pathway as a novel target for cancer therapy. Anticancer Res 28: 3621-3630, 2008. 